\title{
Results from the experimental campaign of the AGATA Demonstrator at LNL
}

\author{
E. Farnea on behalf of the AGATA Collaboration \\ INFN Sezione di Padova, 35131 Padova, Italy
}

\begin{abstract}
The AGATA Demonstrator Array has recently concluded a two-years campaign of measurements at the Laboratori Nazionali di Legnaro, Italy. In this contribution, the principles of operation of the device and the status of the AGATA project will be reviewed. The performance of the array will be discussed and highlights from a few selected measurements will be presented.
\end{abstract}

\section{Introduction}

In the past 20 years, valuable Nuclear Structure information has been obtained with $\gamma$ spectroscopy techniques, by using arrays of Compton-suppressed hyperpure germanium (HPGe) detectors. These devices rely on the combination of several crystals to obtain full peak efficiencies of the order of a few percent (up to $10 \%$ for arrays such as EUROBALL and GAMMASPHERE [1]). The peak-to-total $(\mathrm{P} / \mathrm{T})$ ratio of the resulting spectra is maximised by collimating the detectors, which limits the scattering of photons in between crystals, and, most importantly, by using veto detectors (Compton-suppression shields) to detect photons which made only a partial energy deposition within the germanium crystals. A major problem with these devices is the Doppler broadening of photons emitted from nuclei in motion with typical recoil velocity of a few percent of the speed of light. In order to limit this effect, the crystals should cover as small a solid angle as possible.

The above described devices are not well suited to the experimental conditions at the planned and under construction radioactive ion beam facilities such as SPES, SPIRAL-2, FAIR. Full peak efficiencies of the order of $30-40 \%$ will be needed to cope with the low beam intensities. In case of fragmentation facilities with high-energy beams, where the nuclei emitting the radiation will move with relativistic velocities, Doppler broadening will dominate the effective energy resolution of the detectors. "Conventional" arrays should be composed of thousands of crystals in order to keep these effects to acceptable values, which would hardly be manageable and, most likely, economically unfeasible.

In the early 2000s an innovative approach to this problem was proposed, namely to use the germanium crystals in position-sensitive mode. This requires using crystals with electrically segmented outer electrodes and digital electronics. The energy and position of each interaction within the crystals is extracted by comparison of the observed signal shapes with a reference set of signals representing the response of the system to a single-interaction event in a grid of known locations inside the crystal. This process is known as signal decomposition or pulse shape analysis, PSA. Once the full set of interaction points seen in all detectors in a specific event is determined, the energy and direction of each photon can be determined (tracked) by means of computer algorithms, which, as a side effect, can also perform efficient Compton suppression, or, in other words, identify and discard partial energy depositions. The full-peak efficiency of a $4 \pi$ tracking array of HPGe detectors, as estimated by realistic Monte Carlo simulations, can as high as $50 \%$, with a $\mathrm{P} / \mathrm{T}$ ratio of $60 \%$. Since each interaction point is determined with a precision of a few millimeters, hence much smaller than the typical crystal size, the Doppler correction can be performed with a much better quality than in case of "conventional" Compton-suppressed arrays.

Presently, the construction of a tracking array inspired by the above mentioned principles is pursued by two projects, both using 36-fold segmented crystals closely packed into multi-crystal cryostat (clusters) and digital electronics sampling all signals at $100 \mathrm{Ms} / \mathrm{s}$ with a resolution of 14 bits. The USbased array GRETA [2] will be built out of 120 crystals, arranged into 30 quadruple clusters, while the 


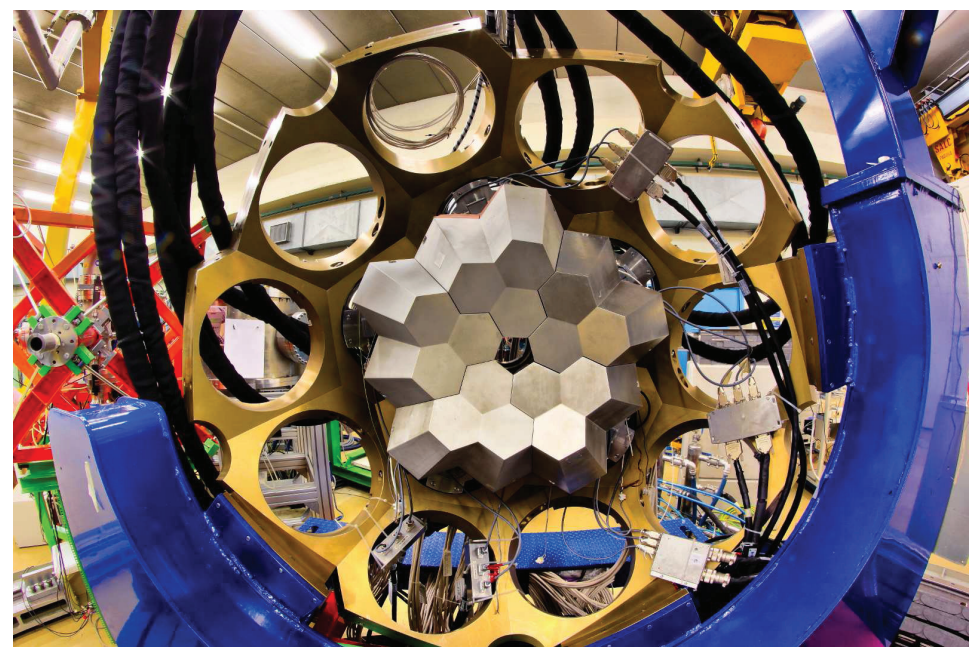

Fig. 1: Photo of the AGATA Demonstrator Array installed at the target point of the PRISMA magnetic spectrometer at the Laboratori Nazionali di Legnaro.

European array AGATA [3] will be composed of 180 crystals, arranged into 60 triple clusters. The two projects are very ambitious, technically challenging and, clearly, economically compelling. Therefore, they will be completed in stages, starting with the realization of a sub-set of the full array to "demonstrate" the soundness and feasibility of the chosen technical solutions. A key point in both projects is the capability of reducing the huge data flow $(200 \mathrm{MB} / \mathrm{s} /$ segment, i.e. $8 \mathrm{~GB} / \mathrm{s} /$ crystal) to values which can be handled by the available computer technology. For the full arrays, it will be impossible to store the original digitized data, implying that pulse shape analysis and $\gamma$-ray tracking should be performed in real time, and only the final results of the tracking process should be stored.

\section{The AGATA Demonstrator at LNL}

The AGATA Demonstrator consists of five AGATA triple clusters, arranged in the compact configuration shown in the Figure 1. Given that this configuration lacks spherical symmetry and that, contrary to conventional arrays, the detectors are not bound to a single source-detector distance, the performance of the AGATA Demonstrator depends on its position relative to the source. According to the Monte Carlo simulations reported in [4], the full peak efficiency of the array for $1 \mathrm{MeV}$ photons ranges between $3 \%$ and $7 \%$ with a $\mathrm{P} / \mathrm{T}$ ratio close to $60 \%$. These values, later confirmed by the experimental data, are comparable to existing arrays of Compton-suppressed detectors. Therefore, in the initial phase, the emphasis to prove that AGATA will be a much superior device is put on the quality of the Doppler correction. This means that the AGATA Demonstrator is best exploited in combination with devices to track the incoming beam or the recoiling nuclei. The AGATA Demonstrator has been first installed at the Laboratori Nazionali di Legnaro (LNL), Italy, at the target point of the large-acceptance magnetic spectrometer PRISMA [5]. The initial goal of the campaign of measurements was to prove that indeed PSA and $\gamma$-ray tracking could be successfully performed in real time, on the most demanding conditions achievable in a low-energy stable-beam facility, i.e. with reactions with velocities of the $\gamma$-emitting products up to $\beta 10 \%$ and with relatively high-intensity beams. Once this was achieved, the Demonstrator was used for physics experiments, mostly in coupled operation with PRISMA and with the array of MCP detectors DANTE [6] to study moderately neutron-rich nuclei populated by multi-nucleon transfer or deep inelas- 


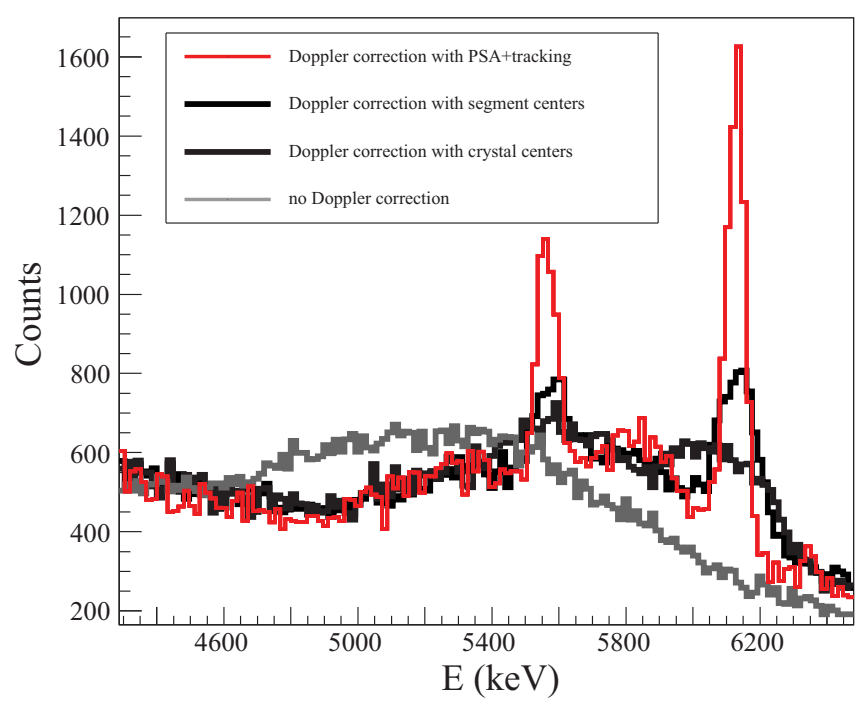

Fig. 2: Quality of the Doppler correction obtained with the AGATA Demonstrator coupled to the segmented silicon detectors of the TRACE project. Spectra obtained in coincidence with a ${ }^{16} \mathrm{O}$ nucleus detected within TRACE are shown, performing Doppler correction under different conditions. See text for details.

tic collisions with the stable beams delivered by the Tandem-PIAVE-ALPI accelerator complex. AGATA was also successfully coupled with other complementary detectors in order to exploit experimental possibilities such as direct, Coulomb excitation or fusion-evaporation reactions. The technical details on the installation of the Demonstrator at LNL are discussed in [7].

\section{Performance of the AGATA Demonstrator at LNL}

The commissioning campaign of the AGATA Demonstrator at LNL was carried out during 2009, through a series of source and in-beam tests. The overall performance of the device was quite satisfactory and has been the subject of technical reports, see for instance [8] or [9]. In the present contribution, we will just discuss the overall quality of the Doppler correction. The spectra shown in Figure 3 were obtained with the ${ }^{17} \mathrm{O}(340 \mathrm{MeV})+{ }^{208} \mathrm{~Pb}$ reaction, where the projectile-like ${ }^{16} \mathrm{O}$ nuclei were detected in the $4 \mathrm{~mm}$ x $4 \mathrm{~mm}$ pads of the silicon detectors of TRACE [7]. In this particular example, Doppler correction was performed using the velocity corresponding to the scattering of a ${ }^{16} \mathrm{O}$ nucleus into the centre of the firing pad. Considering the AGATA crystals as a whole, no peaks are visible, but they clearly start standing out when the segmentation information is used and most importantly when the full information from PSA and tracking is available. The FWHM for the $6130 \mathrm{keV}$ line in ${ }^{16} \mathrm{O}$ is $58 \mathrm{keV}$, which, although far from the intrinsic energy resolution of the germanium detectors, is fully consistent with the kinematics of the reaction, as verified with Monte Carlo simulations. The quality of the Doppler correction reflects the underlying performance of the pulse shape analysis. As shown in [10], the position resolution obtained with PSA techniques is slightly better than $4 \mathrm{~mm}$ FWHM for energies above $1 \mathrm{MeV}$.

\section{The Physics Campaign at LNL}

The AGATA Demonstrator has been exploited in a two-year experimental campaign at the Laboratori Nazionali di Legnaro, Italy. A total of 20 PAC-approved measurements were performed, plus 3 in- 


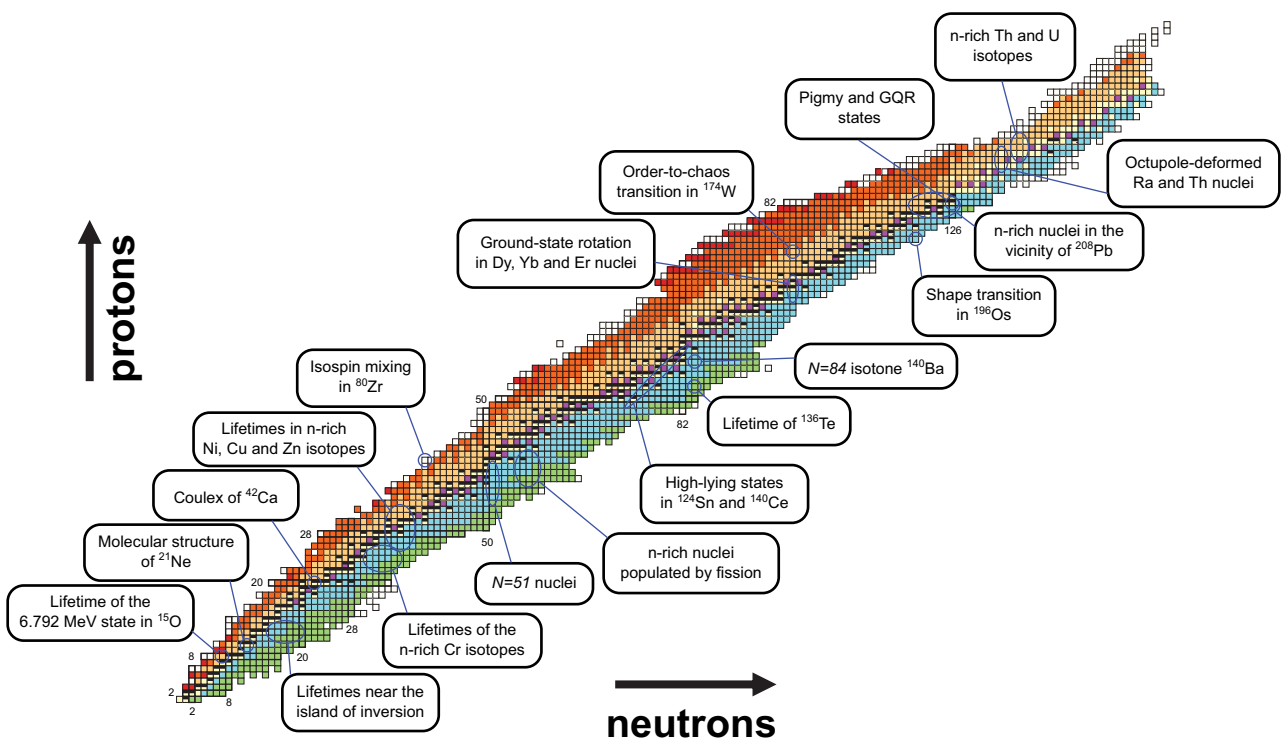

Fig. 3: Visual summary of the first experimental campaign of the AGATA Demonstrator, performed at the Laboratori Nazionali di Legnaro, Italy.

beam tests, for a grand total of 148 days of beam time. A visual summary of the campaign is shown in Figure 4 . The campaign has focused mainly on the study of moderately neutron-rich nuclei populated via multi-nucleon transfer or deep-inelastic reactions in order to best exploit the possibilities offered by the coupling with PRISMA. However, the proton-rich side of the nuclides chart has been explored as well by coupling AGATA with complementary devices such as the silicon detectors of TRACE or the scintillators of HELENA [11] and HECTOR+ [12]. Given the novelty and relative complexity of the PSA and $\gamma$-ray tracking methods, the experiments are still in the analysis phase. Therefore, in this contribution we will just discuss a few preliminary results.

\subsection{Lifetime measurement in $\mathrm{Zn}$ isotopes}

A large fraction of the AGATA-PRISMA experiments at Legnaro aimed at measuring transition probabilities of neutron-rich nuclei populated in multi-nucleon transfer reactions, applying the Recoil Distance Doppler Shift method [13] with the differential plunger device developed at IKP Köln [7]. As a matter of fact, the technique was previously tested at LNL during the CLARA-PRISMA campaign [14]. As an example, we report here on an experiment aimed at studying the onset of collectivity in the zinc isotopes, which was performed with the ${ }^{76} \mathrm{Ge}(577 \mathrm{MeV})+{ }^{238} \mathrm{U}$ reaction. The systematics of $\mathrm{B}\left(\mathrm{E} 2 ; 2^{+}\right.$ $\rightarrow 0^{+}$) for the even neutron-rich zinc isotopes, measured with Coulomb excitation experiments at the REX-ISOLDE facility [15], suggest that collectivity rapidly develops at $N=40$ when moving away from ${ }^{68} \mathrm{Ni}$, which shows instead a doubly-magic nature as suggested by the high excitation energy for its first excited state, together with a low B(E2; $\left.2^{+} \rightarrow 0^{+}\right)$value. Since the statistics in these experiments was not sufficient to extract the angular distribution of the ejectiles, an independent evaluation of the $\mathrm{B}(\mathrm{E} 2)$ via lifetime measurement is needed to derive the deformation of the involved isotopes. A paper on this measurement is forthcoming [16], here we will just mention that the $\mathrm{B}(\mathrm{E} 2)$ for ${ }^{72} \mathrm{Zn}$, obtained via differential decay curve method [17] analysis, are in agreement with known values from the literature, while the corresponding values for ${ }^{74} \mathrm{Zn}$ are lower than the previously accepted values, thus shifting back 
the maximum of $\mathrm{B}\left(\mathrm{E} 2 ; 2^{+} \rightarrow 0^{+}\right)$for the $\mathrm{Zn}$ chain from $N=44$ to $N=42$.

\subsection{Lifetime measurement in ${ }^{15} \mathrm{O}$}

As a second (and final) example, we report on a lifetime measurement to gather nuclear structure information of astrophysical interest related to the core metallicity of the Sun [18]. Assuming that all the relevant reaction cross sections are known, the carbon and nitrogen content in the center of the Sun can be deduced from the $\mathrm{CNO}$ neutrino fluxes. The overall energy production rate is determined by the slowest reaction of the cycle, namely by the ${ }^{14} \mathrm{~N}(\mathrm{p}, \gamma){ }^{15} \mathrm{O}$ reaction. In particular, the width of the sub-threshold resonance corresponding to the $6.79 \mathrm{MeV}$ excited state in ${ }^{15} \mathrm{O}$ plays a crucial role in the evaluation of the total astrophysical S-factor at zero energy, as discussed in [19]. To improve the accuracy of such value, a new direct measurement of the lifetime of this level was performed by means of the Doppler Shift Attenuation Method (DSAM) [13]. The technique was pushed to the fs range in which the lifetime of the level of interest is expected to lie by populating the excited level in ${ }^{15} \mathrm{O}$ in inverse kinematics, producing the ${ }^{14} \mathrm{~N}$ beam at $32 \mathrm{MeV}$ energy with the Tandem accelerator and using a deuterated gold target. The Demonstrator, used in standalone mode, consisted of 4 triple clusters. The details and the results of the analysis will be the subject of a forthcoming publication [20], here we will just mention that it was possible to construct energy versus angle matrices and to perform DSAM with an almost continuous angular distribution by exploiting the position-sensitive capabilities of the AGATA crystals. The level lifetime will be extracted through a comparison with detailed Monte Carlo simulations of the experimental setup and of the reaction process, which is mostly direct nucleon transfer with an admixture of fusion-evaporation. The method was validated with a known level in ${ }^{15} \mathrm{~N}$, populated during the same run. The preliminary results on ${ }^{15} \mathrm{O}$ point to a very short lifetime of the order of $1 \mathrm{fs}$, in agreement with the literature [21].

The author would like to thank all of the AGATA collaboration for the support during the experimental campaign at Legnaro. In particular, the author would like to thank Corinne Louchart and Caterina Michelagnoli for sharing their preliminary results.

\section{References}

[1] J. Eberth and J. Simpson, Prog. Part. Nucl. Phys. 60, 283 (2008).

[2] M.A. Deleplanque et al., Nucl. Instr. and Meth. A430, 292 (1999).

[3] S. Akkoyun et al., Nucl. Instr. and Meth. A668, 26 (2012).

[4] E. Farnea et al., Nucl. Instr. and Meth. A621, 331 (2010).

[5] A.M. Stefanini et al., Nucl. Phys. A701, 217c (2002); S. Szilner et al., Phys. Rev. C76, 024604 (2007).

[6] A. Gottardo et al., Nucl. Phys. A805, 606 (2008).

[7] A. Gadea et al., Nucl. Instr. and Meth. A654, 88 (2011).

[8] F. Recchia et al., INFN-LNL-234, 60 (2011).

[9] B. Bruyneel et al., INFN-LNL-234, 64 (2011).

[10] P.-A. Söderström et al., Nucl. Instr. and Meth. A638, 96 (2011).

[11] M. Mattiuzzi et al., Nucl. Phys. A612, 262 (1997).

[12] R. Nicolini et al., Nucl. Instr. and Meth. A582, 554 (2007); F.G.A. Quarati et al., Nucl. Instr. and Meth. A629, 157 (2011).

[13] T.K.Alexander, J.S.Forster, in: M. Baranger, E. Vogt (Eds.), Advances in Nuclear physics, Vol.10, Plenum Publishing Corporation (1978) 
[14] J.J. Valiente-Dobón et al., Phys. Rev. Lett. 102, 242502 (2009).

[15] J. Van de Walle et al., Phys. Rev. Lett. 99, 142501 (2007).

[16] C.Louchart et al., in preparation (2012)

[17] A. Dewald et al., Z. Phys. A334, 163 (1989).

[18] A.M. Serenelli et al., Astrop. Journ. Lett. 705, L123 (2009).

[19] C. Broggini et al., Annu. Rev. Nucl. Sci., 60, 53 (2010) and references therein.

[20] C.Michelagnoli et al., in preparation (2012)

[21] C.Michelagnoli et al., proceedings of the $11^{\text {th }}$ International Symposium on Origin of Matter and Evolution of Galaxies, Wako, Saitama, Japan, 2011 (in press). 